\title{
Estimando parâmetros cosmológicos a partir de dados observacionais
}

\author{
Estimating cosmological parameters from observational data
}

\author{
Gival Pordeus da Silva Neto*1,2
}

\author{
${ }^{1}$ Departamento de Física Teórica e Experimental, Universidade Federal do Rio Grande do Norte, Av. Sen. Salgado Filho, 3000 \\ - Lagoa Nova, CEP. 59078-970, Natal, RN, Brasil \\ ${ }^{2}$ CAIC José Joffily, Secretaria de Estado da Educação, Governo da Paraíba, R. José Marques Ferreira, 100 - Malvinas, CEP. \\ 58432-545, Campina Grande, PB, Brasil
}

Recebido em 05 de Agosto, 2017. Revisado em 04 de Novembro, 2017. Aceito em 09 de Novembro, 2017.

\begin{abstract}
Neste trabalho são realizadas análises estatísticas capazes de estimar significativamente os valores atuais de alguns dos principais parâmetros cosmológicos, em particular, a taxa de expansão do Universo $H_{0}$ (Constante de Hubble), a densidade de matéria $\Omega_{0, m}$, a densidade de energia escura $\Omega_{0, \Lambda}$, a idade do Universo $t_{0}$ e o parâmetro de desaceleração $q_{0}$. Além disso, são abordados observáveis importantes como as Oscilações Acústicas dos Bárions e o Parâmetro de Hubble, além do Método Qui-quadrado e alguns conceitos e equações básicas da Cosmologia. A partir da descrição detalhada apresentada, objetiva-se dar uma ideia para os não especialistas de como estes valores podem ser obtidos a partir de dados observacionais disponíveis na literatura. Por último, é realizado uma comparação dos resultados obtidos neste trabalho com os obtidos por grandes grupos internacionais como a Planck Collaboration e o WMAP. Os resultados são bem restritivos e em acordo com os estimados pela Planck Collaboration e o WMAP.

Palavras-chave: Modelo $\Lambda$ CDM, Parâmetros Cosmológicos, Constante de Hubble, Oscilações Acústicas dos Bárions, Método Qui-quadrado.
\end{abstract}

In this work, it is performed statistical analyzes capable of estimating the current value of some of the main cosmological parameters, in particular, the rate of expansion of the Universe $H_{0}$ (Hubble Constant), the density of matter $\Omega_{0, m}$, the density of dark energy $\Omega_{0, \Lambda}$, the age of the Universe $t_{0}$ and the deceleration parameter $q_{0}$. In addition, it is discussed important observables as the Baryon Acoustic Oscillations and the Hubble Parameter, besides the Chi-square Method and some basic concepts and equations of Cosmology. From the detailed description presented it is intended to give an idea to non-specialists of how these values can be obtained from observational data available in the literature. Lastly it is performed a comparison of the results obtained in this work with those obtained by large international groups as the Planck Collaboration and the WMAP. The results are very restrictive and in agreement with those estimated by Planck Collaboration and the WMAP.

Keywords: $\Lambda$ CDM Model, Cosmological Parameters, Hubble Constant, Baryon Acoustic Oscillations, Chi-square Method.

\section{Introdução}

Hoje, a Cosmologia pode ser definida como sendo a ciência que estuda a origem, estrutura e a evolução do Universo. Seu principal objetivo é entender como o Universo se formou, porque possui as características observadas hoje e saber como o mesmo será no futuro.

O surgimento da Cosmologia moderna se deu a partir do desenvolvimento da teoria da Relatividade Geral (RG), publicada por Einstein em $1915^{1}$ Essa teoria é fundamental para construir um modelo cosmológico, pois é a teoria gravitacional que melhor lida com os paradoxos

\footnotetext{
*Endereço de correspondência: givalpordeus@hotmail.com

${ }^{1}$ Detalhes sobre a história da Cosmologia nesses últimos 100 anos, seus maiores avanços e suas principais questões ainda em aberto, são encontrados na referência 1 .
}

associados a um espaço que se estende ao infinito e que melhor explica as observações e fenômenos estudados até o momento.

Por sua vez, a Cosmologia Observacional é uma subárea da Cosmologia que se desenvolveu bastante nos últimos anos, motivada principalmente pela grande evolução tecnológica dos instrumentos utilizados em observações astronômicas. Tal desenvolvimento foi essencial para tornar a Cosmologia uma ciência de precisão, e assim, acabar com o seu caráter puramente especulativo e filosófico. Um dos principais objetivos da Cosmologia Observacional é medir o valor dos mais diversos parâmetros cosmológicos a partir de observações astronômicas.

Atualmente, o modelo cosmológico que fornece melhor ajuste com os dados observacionais é o Modelo $\Lambda \mathrm{CDM}$ (Lambda Cold Dark Matter), também conhecido como 
Modelo Padrão ou Modelo de Concordância Cósmica. Esse modelo tem como base três pilares: a Expansão do Universo, a Nucleossíntese Primordial e a Radiação Cósmica de Fundo.

É uma prática padrão em Cosmologia, especificar modelos cosmológicos através de alguns parâmetros. Daí, para obter-se a versão do modelo que melhor descreve o Universo, é necessário determinar observacionalmente o valor destes parâmetros. Quanto mais restritiva e acurada for a determinação, melhor e mais precisa será a descrição do Universo fornecida por este modelo.

Neste trabalho foram realizadas análises estatísticas usando dados observacionais do Parâmetro de Hubble $(\mathrm{DO} H(z))$ e do Pico das Oscilações Acústicas dos Bárions (BAO) em um Modelo $\Lambda$ CDM Plano, objetivando estimar os valores de alguns dos principais parâmetros deste modelo, a saber, $H_{0}, \Omega_{0, m}, \Omega_{0, \Lambda}, q_{0}$ e $t_{0}$. Além disso, com uma descrição detalhada e objetiva da análise, é esperado que mesmo os profissionais que não tenham afinidade com tais técnicas, compreendam e possam ter uma ideia de como é possível estimar os valores dessas e de outras grandezas.

\section{Cosmologia: fundamentos teóricos}

Nesta seção, são apresentados de forma sucinta e objetiva, os fundamentos teóricos básicos da Cosmologia padrão moderna, necessários para a compreensão adequada do trabalho.

\subsection{A expansão do Universo e a Constante de Hubble}

No início do século XX, acreditava-se que o Universo era estático. Contrapondo-se a essa concepção, Alexander Friedmann em 1922 e Georges Lemâitre em 1927, obtiveram soluções para equações de Einstein que descreviam modelos de Universo dinâmico. No entanto, a concepção de um Universo estático só foi abandonada no início da década de 1930. Principalmente após os trabalhos do astrônomo americano Edwin P. Hubble [2,3, onde foi possível concluir que há um aumento sistemático da velocidade de recessão (afastamento) das galáxias com a distância, como sugere a Figura 1. Essa figura permite concluir que o Universo está em expansão, pois se ele fosse estático, estatisticamente devia-se observar tanto velocidades positivas (de recessão) como negativas (de aproximação) [4]. Em seu trabalho [2], Hubble propôs uma relação linear entre a velocidade de recessão e a distância, ou seją2

$$
v=H_{0} d
$$

onde $H_{0}$ é uma constante de proporcionalidade, chamada de Constante de Hubble. Seu valor representa a taxa atual de expansão do Universo. Por motivo de conveniência, usualmente a Contante Hubble é expressa em termo de um parâmetro adimensional $h$,

$$
H_{0} \equiv h \cdot 100 \mathrm{~km} \cdot s^{-1} \cdot \mathrm{Mpc}^{-1} \text {, }
$$

onde, hoje se sabe que $0,60<h<0,90$. Em Cosmologia, medidas de velocidade normalmente são expressas em $\mathrm{km} \cdot \mathrm{s}^{-1}$ e de distância em megaparsec (Mpc), onde $1 \mathrm{Mpc} \simeq 3,26 \cdot 10^{6}$ anos $\mathrm{luz} \simeq 3,08 \times 10^{22} \mathrm{~m}$.

A Constante de Hubble é sem dúvida uma das constantes mais fundamentais da Cosmologia. Ela está relacionada com diversas grandezas cosmológicas, como por exemplo, as distâncias físicas entre objetos astronômicos, a idade do Universo e sua densidade de energia. Portanto, por ser crucial para qualquer modelo cosmológico moderno, é de extrema importância a determinação mais exata possível do seu valor, para assim termos um modelo cosmológico que seja o mais acurado possível.

${ }^{2}$ Para mais informações sobre a descoberta da expansão do Universo e a Lei de Hubble, é sugerida a leitura das referências \begin{tabular}{|l|l|l|l|}
1 & 2 & 4
\end{tabular}

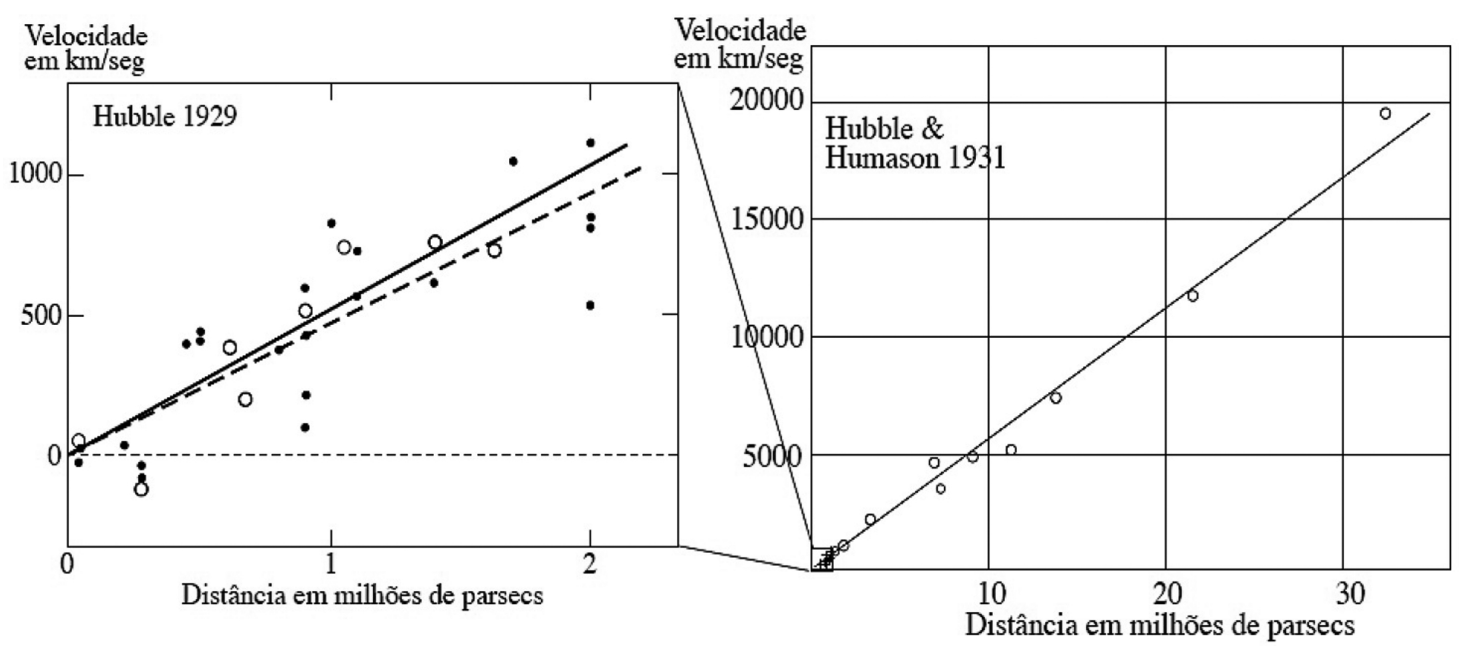

Figura 1: Relação entre a velocidade de recessão e a distância. Esquerda: figura 1 da referência [2]. Direita: figura 5 da referência [3] (extraída de [5]). 
É importante ressaltar que a equação (1), só é válida para "pequenas" distâncias, ou equivalentemente, pequenos desvios para o vermelhd 3 onde a relação $v=c z$ é válida [4]. Por outro lado, a expressão

$$
v_{p}=H(t) d_{p}
$$

conhecida como Lei de Hubble, é sempre válida. A grandeza $v_{p}$ e $d_{p}$ representa a velocidade e distância própria (ou física), respectivamente. Já a quantidade $H(t)$, chamada de Parâmetro de Hubble, representa a taxa de expansão do Universo em um dado tempo $t$, sendo portanto, $H_{0}$ o valor atual de $H(t)$, ou seja, $H_{0} \equiv H\left(t=t_{0}\right)$. Neste contexto a equação (1) é um caso particular da equação (3). Na verdade, a Lei de Hubble é uma consequência da homogeneidade e isotropia do Universo, uma vez que essa é a única lei de expansão compatível com um Universo homogêneo e isotrópiç 4,6 .

O Parâmetro de Hubble é definido em termos do fator de escala da seguinte forma ${ }^{5}$.

$$
H(t) \equiv \frac{\dot{a}(t)}{a(t)} .
$$

O fator de escala, aqui denominado pela letra $a(t)$, é uma quantidade fundamental quando se estuda um Universo que se expande. Ele indica, por exemplo, como as distâncias cosmológicas variam com o tempo. Assim, através dele, podemos saber o quanto elas eram menores no passado quando comparadas com essas mesmas distâncias medidas hoje. Da equação (4), têm-se que quanto maior for a variação do fator de escala em um certo intervalo de tempo, maior será a taxa de expansão do Universo neste mesmo intervalo.

\subsection{Geometria, dinâmica e os componentes do fluido cosmológico}

As equações básicas da RG são as do campo gravitacional, conhecidas como as equações de campo de Einstein 7,8$]$ :

$$
G_{\mu \nu} \equiv R_{\mu \nu}-\frac{1}{2} g_{\mu \nu} R-\Lambda g_{\mu \nu}=\frac{8 \pi G}{c^{4}} T_{\mu \nu}
$$

onde $G_{\mu \nu}$ é o tensor de Einstein, que contém as propriedades geométricas do espaço-tempo, $T_{\mu \nu}$ é o tensor energia-momento, que representa a distribuição da matéria-energia, $R_{\mu \nu}$ é o tensor de Ricci, $R$ é o escalar de curvatura de Ricci, $G$ é a constante gravitacional, $\Lambda$ é a constante cosmológica e $\frac{8 \pi G}{c^{4}}$ é a constate de Einstein.

Para estudar a dinâmica cósmica, baseada na equação (5), é preciso de antemão determinar a métrica e as componentes do tensor energia-momento. Para obter o tensor energia-momento $T_{\mu \nu}$, supõe-se que o Universo seja preenchido por um fluido perfeito, ou melhor, uma soma

${ }^{3}$ Deslocamento para o vermelho das linhas espectrais, representada pela letra $z$ (ver seção 2.4.

${ }^{4}$ Para mais detalhes ver seção 1.1 da referência 6 .

${ }^{5}$ Neste artigo, usa-se a notação ". " para representar a derivada da grandeza com relação ao tempo, ou seja: $\dot{a} \equiv \frac{d a}{d t}$ e $\ddot{a} \equiv \frac{d^{2} a}{d t^{2}}$. de fluidos perfeitos (radiação, matéria, entre outros), cujo o tensor energia-momento é 9 :

$$
T_{\mu \nu}=\sum_{i}\left(\rho_{i}+\frac{p_{i}}{c^{2}}\right) u_{\mu} u_{\nu}-\sum_{i} p_{i} g_{\mu \nu}
$$

onde $\rho_{i}$ é a densidade do fluido, $p_{i}$ é a pressão do fluido, $g_{\mu \nu}$ é o tensor métrico (ver equação (7)) e $u_{\mu}=\left(c^{2}, 0,0,0\right)$ é a quadri-velocidade medida no referencial comóvel. Devido a homogeneidade e isotropia, a pressão $p_{i}$ e a densidade $\rho_{i}$ só dependem do tempo.

Para determinar a métrica, Einstein fez uso de um princípio simplificador chamado de Princípio Cosmológico. Este considera que em escala suficientemente grande o Universo é espacialmente homogêneo e isotrópico. Homogeneidade é a afirmação de que o Universo tem a mesma aparência em cada ponto, enquanto isotropia é a afirmação de que o Universo tem a mesma aparência em todas as direções [10]. O Princípio Cosmológico permaneceu apenas uma hipótese inteligente até que dados empíricos sólidos confirmando a homogeneidade e isotropia em grande escala fossem finalmente obtidos no final do século XX 11]. Pesquisas sugerem que o Universo passa a ser realmente homogêneo e isotrópico em escalas superiores a $100 \mathrm{Mpc}$ 6, 12]; em escalas menores existem grandes inomogeneidades, como galáxias, aglomerados e superaglomerados de galáxias.

Espaços homogêneos e isotrópicos possuem o maior grupo de simetria possível, restringindo fortemente a geometria admissível para esses espaços. A métrica mais geral que descreve um Universo em expansão e que satisfaz estas restrições impostas pelo Princípio Cosmológico, é a métrica de Friedmann-Robertson-Walker (FRW), que expressa em coordenadas esféricas comóveis possui a seguinte forma 9,13 :

$$
\begin{aligned}
d s^{2} & =g_{\mu \nu} d x^{\mu} d x^{\nu}=c^{2} d t^{2}-a^{2}(t) \\
& \times\left[\frac{d r^{2}}{1-k r^{2}}+r^{2} d \theta^{2}+r^{2} \operatorname{sen}^{2} \theta d \phi^{2}\right],
\end{aligned}
$$

onde $x^{\mu}=\left(x^{0}=c t, x^{1}=r, x^{2}=\theta, x^{3}=\phi\right)$ e $g_{\mu \nu}$ é o tensor métrico, $g_{\mu \nu} \doteq \operatorname{diag}\left(c^{2},-\frac{a^{2}}{1-k r^{2}},-a^{2} r^{2},-a^{2} r^{2} \sin ^{2}(\theta)\right)$.

Essa métrica é caracterizada por duas quantidades: o fator de escala $a(t)$ e a constante $k$, que determina se o Universo é espacialmente plano $(k=0)$, esférico $(k=1)$ ou hiperbólico $(k=-1)$ 9,13. A Figura 2 ilustra superfícies bidimensionais com $k=1,0$ e -1 , que pode possibilitar alguma intuição das análogas tridimensionais.

De posse das componentes do tensor de Einstein $G_{\mu \nu}$ e do tensor energia-momento $T_{\mu \nu}$, é possível resolver a equação (5), que devido o elevado grau de simetria e por muito dos termos se anularem, o cálculo leva apenas à duas equações independentes, que são [9]:

$$
\dot{a}^{2}=\frac{8 \pi G}{3} a^{2} \sum_{i} \rho_{i}+\frac{1}{3} a^{2} c^{2} \Lambda-c^{2} k
$$

$\mathrm{e}$

$$
\frac{\ddot{a}}{a}=-\frac{4 \pi G}{3} \sum_{i}\left(\rho_{i}+3 \frac{p_{i}}{c^{2}}\right)+\frac{1}{3} c^{2} \Lambda .
$$



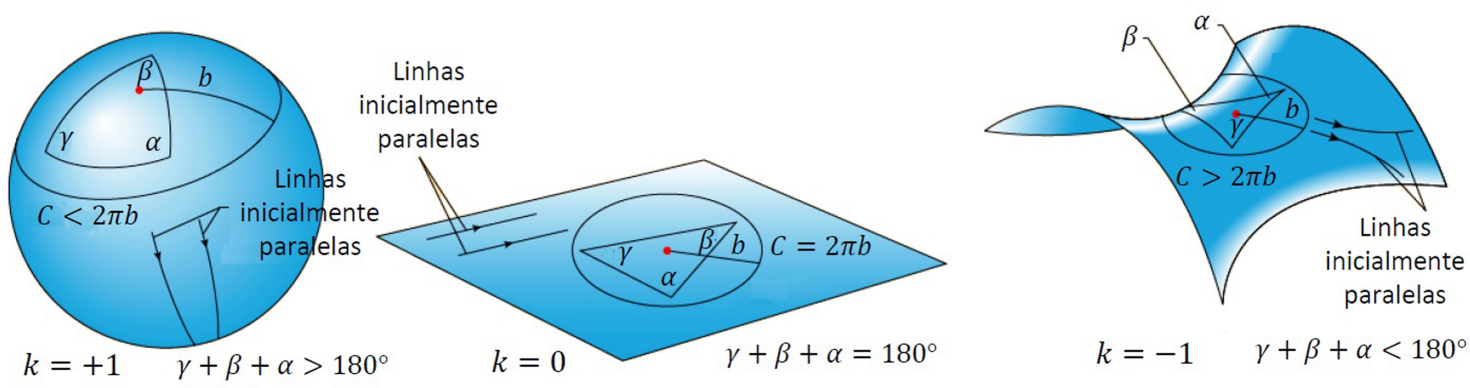

Figura 2: Três superfícies bidimensionais: a esférica tem $k=1$, a plana tem $k=0$ e a em forma de sela tem $k=-1$. Em superfícies curvas $(k \neq 0)$ a soma dos ângulos de um triângulo não é igual a $180^{\circ}$, a circunferência de um círculo não é igual a $2 \pi$ vezes o raio e as geodésicas que se iniciam paralelas, não permanecem paralelas (adaptado da referência [15]).

Essas duas equações governam a dinâmica do Universo, determinando a evolução temporal do fator de escala $a(t)$, e são conhecidas como as equações de FriedmannLemaître. No caso $\Lambda=0$, essas equações são muitas vezes chamadas simplesmente de equações de Friedmann.

Assumindo que os fluidos são não interagentes, é possível obter das equações (8) e (9), uma importante relação que expressa a conservação da matéria-energia, dada por:

$$
\dot{\rho}_{i}+3 \frac{\dot{a}}{a}\left(\rho_{i}+\frac{p_{i}}{c^{2}}\right)=0 .
$$

Em cosmologia, é habitual assumir que cada componente do fluido cosmológico possui uma equação de estado da forma $p_{i}=\omega_{i} \rho_{i} c^{2}[13$. Daí, substituindo essa relação na equação 10 e supondo $\omega_{i}$ constante, é possível mostrar que a densidade de cada fluido evolui independentemente, da seguinte forma:

$$
\rho_{i}(t)=\rho_{0, i}\left(\frac{a(t)}{a_{0}}\right)^{-3\left(1+\omega_{i}\right)}
$$

onde, $\omega_{i}$ é o parâmetro da equação de estado, $\rho_{0, i}$ e $a_{0}$ representam os valores atuais da i-ésima componente constituinte e do fator de escala, respectivamente.

Em geral, os modelos cosmológicos modernos consideram o Universo constituído por três componentes: matéria, radiação e constante cosmológica [9]. A componente da matéria é formada por dois tipos diferentes, a matéria bariônica, constituída basicamente de bárions (prótons, nêutrons e todos os corpos formados deles) e a matéria escura ${ }^{6}$ uma forma mais exótica de matéria constituída por partículas fundamentais, que estão além do modelo padrão da física de partículas. A componente de radiação, por sua vez, inclui os fótons e também partículas ultra-relativísticas com massas não nulas, como os neutrinos. Já a constante cosmológica, é normalmente interpretada como um componente do Universo que tem $\omega=-1$ e, portanto, $p_{\Lambda}=-\rho_{\Lambda} c^{2} 14$.

Por serem bem diluídas as componentes da matéria não-relativística obedecem a lei dos gases perfeitos,

$$
p_{i}=\frac{\rho_{i}}{\mu_{i}} K T_{i}
$$

${ }^{6}$ É chamada de escura porque não emite e nem interage de forma significativa com a radiação, tornado-se assim, impossível "vê-la". onde $\mu_{i}$ é a média das massas das partículas da i-ésima componente. Além disso, a temperatura $T$ e a média quadrática das velocidades térmicas $\left\langle v_{i}^{2}\right\rangle$ de tais componentes, estão associadas pela relação $3 K T_{i}=\mu_{i}\left\langle v_{i}^{2}\right\rangle$ 14. Portanto, lembrando que foi suposto a equação de estado $p_{i}=\omega_{i} \rho_{i} c^{2}$, têm-se que:

$$
p_{i}=\frac{\rho_{i}}{3}\left\langle v_{i}^{2}\right\rangle \Rightarrow \omega_{i}=\frac{\left\langle v_{i}^{2}\right\rangle}{3 c^{2}} .
$$

Logo, para as componentes da matéria não-relativística, as quais $\left\langle v_{m}^{2}\right\rangle \ll c^{2}$, obtêm-se que $\omega_{m} \simeq 0$.

Por outro lado, a pressão dos fóton $\mathbf{7}^{7}$ ou de qualquer outro componente relativística, é dada por $p_{r}=\rho_{r} c^{2} / 3$, $\operatorname{logo}, \omega_{r}=1 / 3$. Uma componente de partículas com massa mas altamente relativística $\left(\left\langle v_{m}^{2}\right\rangle \sim c^{2}\right)$, também terá $\omega_{r}=1 / 3$, já uma ligeiramente relativística $(0<$ $\left.\left\langle v_{m}^{2}\right\rangle<c^{2}\right)$, terá $0<\omega<1 / 3$.

Na Tabela 1] é apresentado o valor de $\omega$ e a dependência explícita da densidade com o fator de escala para cada componente do fluido cósmico. Dessa dependência é possível concluir que no passado distante, onde $a(t) / a_{0} \ll 1$, a dinâmica do Universo foi dominada pela radiação $\left(\rho \propto a(t)^{-4}\right)$, logo depois, dominada pela matéria $\left(\rho \propto a(t)^{-3}\right)$ e atualmente uma dinâmica dominada pela constante cosmológica.

Tabela 1: Valor do parâmetro da equação de estado $\omega$ e lei de evolução da densidade $\rho(t)$ para os principais componentes do fluido cósmico.

\begin{tabular}{ccc}
\hline Componentes & $\omega$ & $\rho(t)$ \\
\hline Matéria (bariônica e escura) & 0 & $\rho_{0, m}\left(\frac{a(t)}{a_{0}}\right)^{-3}$ \\
Radiação (fótons e neutrinos) & $1 / 3$ & $\rho_{0, r}\left(\frac{a(t)}{a_{0}}\right)^{-4}$ \\
Constante cosmológica & -1 & $\rho_{0, \Lambda}$ \\
\hline
\end{tabular}

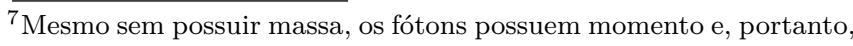
exercem pressão.
} 


\subsection{Parâmetros cosmológicos}

Ao definir $\rho_{\Lambda} \equiv \Lambda c^{2} / 8 \pi G$ e assumir

$$
\rho_{\text {total }}=\sum_{j} \rho_{j}
$$

sendo $j \equiv r, m$ e $\Lambda$, é possível mostrar, substituindo a equação (4) na (8), que:

$$
\frac{c^{2} k}{a^{2} H(t)^{2}}=\left(\frac{8 \pi G}{3 H(t)^{2}} \rho_{\text {total }}-1\right) \text {. }
$$

Nota-se desta expressão que o Universo é espacialmente plano (ou seja, $k=0$ ) somente se a densidade total $\rho_{\text {total }}$ for igual a uma densidade crítica, dada por:

$$
\rho_{\mathrm{crit}}=\frac{3 H(t)^{2}}{8 \pi G}
$$

cujo valor atual é:

$$
\rho_{0, \text { crit }}=\frac{3 H_{0}^{2}}{8 \pi G}=1,878 \cdot 10^{-29} h^{2} \frac{\text { gramas }}{\mathrm{cm}^{3}} .
$$

É conveniente definir, a partir da densidade crítica, parâmetros adimensionais de densidade da seguinte forma:

$$
\Omega_{j}(t) \equiv \frac{\rho_{j}(t)}{\rho_{\text {crit }}}=\frac{8 \pi G}{3 H(t)^{2}} \rho_{j}(t) .
$$

Além disso, é também prática comum definir o parâmetro adimensional de densidade de curvatura como

$$
\Omega_{k} \equiv-\frac{c^{2} k}{a^{2} H(t)^{2}} .
$$

Assim, pode-se escrever a equação 15 em termos dos parâmetros adimensionais de densidade, como segue:

$$
\Omega_{k}=1-\Omega_{\text {total }} \Rightarrow \Omega_{k}+\Omega_{r}+\Omega_{m}+\Omega_{\Lambda}=1 .
$$

Da equação (20), é possível concluir que:

$$
\begin{aligned}
& \Omega_{\text {total }}=1 \Rightarrow \Omega_{k}=0 \Leftrightarrow \text { plano }(k=0) \\
& \Omega_{\text {total }}>1 \Rightarrow \Omega_{k}<0 \Leftrightarrow \text { esférico }(k=1) \\
& \Omega_{\text {total }}<1 \Rightarrow \Omega_{k}>0 \Leftrightarrow \text { hiperbólico }(k=-1) \text {. }
\end{aligned}
$$

Por outro lado, substituindo as equações (11) e (17) na equação 18, obtêm-se:

$$
\Omega_{j}(t)=\Omega_{0, j} \frac{H_{0}^{2}}{H(t)^{2}}\left(\frac{a(t)}{a_{0}}\right)^{-3\left(1+\omega_{j}\right)} .
$$

Fazendo uso desta equação juntamente com as informações da Tabela 1 e da equação 20 , é possível mostrar que:

$$
\begin{aligned}
E(\mathbf{p} ; t) & \equiv \frac{H(t)}{H_{0}}=\left[\Omega_{0, k}\left(\frac{a(t)}{a_{0}}\right)^{-2}+\Omega_{0, r}\left(\frac{a(t)}{a_{0}}\right)^{-4}\right. \\
& \left.+\Omega_{0, m}\left(\frac{a(t)}{a_{0}}\right)^{-3}+\Omega_{0, \Lambda}\right]^{\frac{1}{2}}
\end{aligned}
$$

onde, $E(\mathbf{p} ; t)$ é o parâmetro adimensional de Hubble e $\mathbf{p} \equiv \Omega_{0, l}(l \equiv k, r, m$ ou $\Lambda)$. É importante notar que toda a Cosmologia está contida em $E(\mathbf{p} ; t)$.

Geralmente, o estudo da aceleração do Universo em modelos cosmológicos é realizado através da definição do parâmetro de desaceleração $q(t)$, definido da seguinte forma:

$$
q(t) \equiv-\frac{a \ddot{a}}{\dot{a}^{2}}=-\frac{a^{2}}{\dot{a}^{2}} \frac{\ddot{a}}{a}=-\frac{1}{H^{2}} \cdot \frac{\ddot{a}}{a}
$$

usando a equação (9) e alguns dos parâmetros já apresentados, é possível mostrar que 8

$$
\begin{aligned}
q(t) & =\frac{1}{2 \rho_{\text {crit }}} \sum_{i} \rho_{i}\left(1+3 \omega_{i}\right)-\Omega_{\Lambda} \\
& =\frac{\Omega_{m}}{2}+\Omega_{r}-\Omega_{\Lambda} .
\end{aligned}
$$

Para o tempo presente $t=t_{0}$, torna-se:

$$
q_{0}=\frac{\Omega_{0, m}}{2}+\Omega_{0, r}-\Omega_{0, \Lambda} .
$$

Sendo assim, a taxa de expansão do Universo é constante se $q_{0}=0$, desacelerada se $q_{0}>0$, e acelerada se $q_{0}<0$.

\subsection{O desvio para o vermelho cosmológico}

Quantitativamente o desvio para o vermelho de uma linha espectral é definido da seguinte forma: $z \equiv\left(\lambda_{0}-\lambda\right) / \lambda$, onde $\lambda_{0}$ é o comprimento de onda medido ao atingir o observador e $\lambda$ é o comprimento de onda emitido, ou seja, o medido na fonte.

O desvio para o vermelho cosmológico é uma consequência da expansão do espaço, devido o mesmo causar um alongamento no comprimento de onda da radiação (ver Figura 3), enquanto esta está em trânsito entre o ponto de emissão e o de observação. Esse alongamento ocorre de forma progressiva e varia proporcionalmente ao fator de escala, $\lambda \propto a$ [16]. Com isso, têm-se $\lambda_{0} / \lambda=a\left(t_{0}\right) / a(t)$, que junto com a definição de $z$, permite obter uma importante relação entre o desvio para o vermelho cosmológico e o fator de escala ${ }^{9}$

$$
1+z=\frac{a\left(t_{0}\right)}{a(t)}
$$

onde $a(t)$ e $a\left(t_{0}\right)$ é o valor do fator de escala no momento da emissão $t$ e da observação $t_{0}$, respectivamente. Em um Universo em expansão, têm-se $a\left(t_{0}\right)>a(t)$, resultando em um $z>0$, ou seja, um desvio para o vermelho. Caso contrário, em um Universo em contração, têm-se $a\left(t_{0}\right)<$ $a(t)$ resultando em um $z<0$, ou seja, um desvio para o azul. 
Momento da emissão:

$$
a(t)=a\left(t_{e}\right)
$$

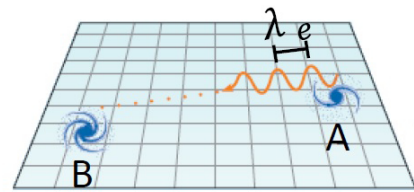

Momento da observação:

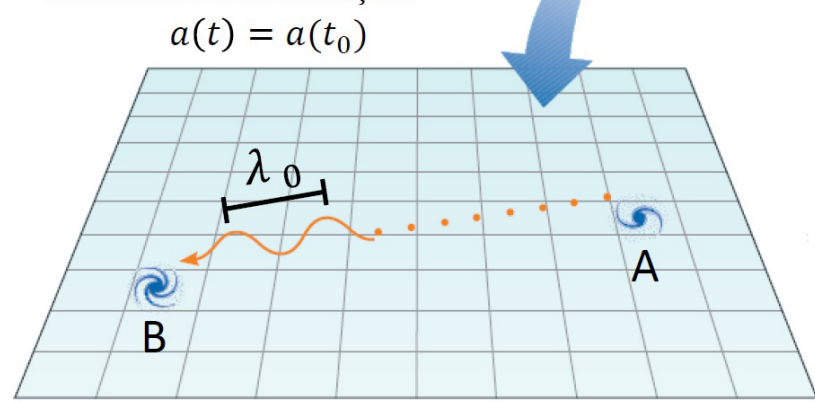

Figura 3: Uma visão esquemática do alongamento do comprimento de onda da radiação enquanto a mesma se propaga da galáxia $A$ à $B$ (adaptado da referência [15]).

\subsection{Idade do Universo e o tempo retrospectivo}

Fazendo uso da equação (26), pode-se expressar $H(t)$ e $E(\mathbf{p} ; t)$ como funções de $z$. Além disso, da mesma têm-se $a_{0} d a=-(1+z)^{-2} d z=-a^{2} d z$, e portanto,

$$
\frac{c d t}{a}=\frac{c d t}{d a} \frac{d a}{a}=\frac{c d a}{H(t) a^{2}} \Rightarrow d t=-\frac{a}{a_{0}} \frac{d z}{H(z)} .
$$

A partir dessa relação, pode-se obter uma expressão que represente a idade do Universo em um $z$ qualquer, ou seja:

$$
\begin{aligned}
t(z) & =\int_{0}^{t(z)} d t=-\int_{\infty}^{z} \frac{a}{a_{0}} \frac{d z^{\prime}}{H\left(z^{\prime}\right)} \\
& =\int_{z}^{\infty} \frac{d z^{\prime}}{\left(1+z^{\prime}\right) H\left(z^{\prime}\right)} .
\end{aligned}
$$

A idade total do Universo $t_{0}$, é obtida tomando $z=0$ na equação anterior,

$$
t_{0}=\frac{1}{H_{0}} \int_{0}^{\infty} \frac{d z^{\prime}}{\left(1+z^{\prime}\right) E\left(\mathbf{p} ; z^{\prime}\right)} .
$$

A relação de tempo retrospectivo é definida como a diferença entre a idade do Universo hoje $\left(t_{0}\right)$ e sua idade quando um raio de luz foi emitido em um particular desvio para o vermelho $(t(z))$. Assim, subtraindo a equação (28) da equação (29), têm-se a relação tempo retrospectivo,

$$
t_{L}(z)=t_{0}-t(z)=\frac{1}{H_{0}} \int_{0}^{z} \frac{d z^{\prime}}{\left(1+z^{\prime}\right) E\left(\mathbf{p} ; z^{\prime}\right)} .
$$

\subsection{Modelo $\Lambda$ CDM Plano}

O Modelo $\Lambda$ CDM é considerado o atual modelo padrão da Cosmologia, por ser o modelo mais simples e que melhor se ajusta às observações e aos dados experimentais.
Esse modelo cosmológico considera o Universo dominado por energia escura (na forma de constante cosmológica $\Lambda$ ), matéria escura fria CDM (não relativística) e com uma contribuição de apenas, aproximadamente, 4,9\% de matéria bariônica. Além disso, considera a constante cosmológica o mecanismo causador da expansão acelerada do Universo, muitas vezes interpretada como energia do vácuo, com equação de estado dada por:

$$
p_{\Lambda}=-\rho_{\Lambda} c^{2}=-\frac{\Lambda c^{4}}{8 \pi G} .
$$

O Modelo $\Lambda$ CDM considera desprezível a densidade de energia da radiaçã $\tilde{q}^{10}\left(\Omega_{0, r} \simeq 0\right)$, frente à densidade de matéria e energia escura. Além do mais, considera o Universo com curvatura espacial nula $\left(k=0, \Omega_{0, k}=\right.$ 0 ), como sugerem as observações da Radiação Cósmica de Fundo (WMAP 9 anos: $\Omega_{0, k}=-0,0027_{-0,0038}^{+0,0039}$ [17, Colaboração Planck: $\left|\Omega_{0, k}\right|<0,005$ [18, ambos com $95 \%$ de confiança estatística). Portanto, para um Modelo $\Lambda$ CDM Plano, têm-se que:

$$
\Omega_{\text {total }}=1 \Rightarrow \Omega_{0, m}+\Omega_{0, \Lambda}=1,
$$

$\log$ :

$$
E\left(\Omega_{0, m} ; z\right)=\frac{H(z)}{H_{0}}=\sqrt{\Omega_{0, m}(1+z)^{3}+\left(1-\Omega_{0, m}\right)}
$$

e o parâmetro atual de desaceleração $q_{0}$, torna-se:

$$
q_{0}=\frac{3}{2} \Omega_{0, m}-1 .
$$

Por outro lado, substituindo a equação $(33)$ na 29$]$ e integrando, obtêm-se a seguinte expressão para a idade atual do Universo:

$$
t_{0}=\frac{2}{3 H_{0} \sqrt{1-\Omega_{0, m}}} \ln \left(\frac{1+\sqrt{1-\Omega_{0, m}}}{\sqrt{\Omega_{0, m}}}\right) .
$$

Veja que a expressão de $t_{0}$ só depende de dois parâmetros, $H_{0}$ (ou, equivalentemente $h$ ) e $\Omega_{0, m}$, já a de $q_{0}$ apenas de um, $\Omega_{0, m}$. Assim, uma vez determinados os valores destes parâmetros, é possível estimar também o valor $t_{0}$ e de $q_{0}$.

\section{Método estatístico e dados observacionais}

\subsection{Método Qui-quadrado}

Suponha que uma grandeza $F$, em um determinado modelo, é verdadeiramente expressa por uma função $F^{\text {teo. }}=F\left(\left\{\alpha_{m}\right\}, z\right)$, onde $\left\{\alpha_{m}\right\}$ são os parâmetros deste modelo. Suponha também uma coleção de $N$ dados observacionais/experimentais $\left\{z_{i}, F_{i}^{o b s}, \sigma_{i}\right\}$, onde $F_{i}^{o b s . ~ c o r r e s-~}$ ponde a medida da grandeza $F^{\text {teo. }}$ quando a grandeza $z$ é igual a $z_{i}$ e $\sigma_{i}$ corresponde a incerteza associada a medida

\footnotetext{
${ }^{10}$ Exceto, claro, na era da radiação.
} 
de $F_{i}^{o b s}$. Assim sendo, a estatística de qui-quadrado $\left(\chi^{2}\right)$ é expressa da seguinte forma:

$$
\chi^{2}=\sum_{i=1}^{N}\left(\frac{F\left(\left\{\alpha_{m}\right\}, z\right)-F_{i}^{o b s .}}{\sigma_{i}}\right)^{2} .
$$

O Método Qui-quadrado consiste em minimizar a estatística de qui-quadrado, variando os parâmetros do modelo. Esta técnica baseia-se na premissa de que o modelo é qualitativamente correto, e é ajustado para minimizar (via $\chi^{2}$ ) as diferenças entre o $F^{\text {teo. }}$ e o $F^{o b s}$. Tais diferenças são consideradas como sendo devido somente as flutuações estatísticas [19]. Na prática, busca-se (por meios de computadores) os valores dos parâmetros que minimizam a estatística de qui-quadrado, pois esses são os que maximizam a função densidade de probabilidade $(L)$ de obter a coleção de dados usados, uma vez que esta é:

$$
L\left(\chi^{2}\right) \propto e^{-\frac{1}{2} \chi^{2}}
$$

Uma das melhores características deste método é que ao mesmo tempo que se estima os parâmetros, verificase também a qualidade do ajuste, ou seja, determina se a função ajustada do modelo é verossími Into é feito certificando se o valor do qui-quadrado mínimo $\left(\chi_{\text {mín. }}^{2}\right)$ é próximo do número de graus de liberdade $(\nu)$, que é dado por $\nu=N-m$, onde $m$ é o número de parâmetros livres e $N$ o de dados observacionais. Isto porque o valor mais provável do $\chi^{2}$, obtido pela condição de máximo da função de densidade de probabilidade, é aproximadamente igual ao $\nu$ [20]. Portanto, quanto mais próximo for o valor do $\chi_{\text {mín. }}^{2}$ do valor de $\nu$, melhor é o ajuste do modelo aos dados observacionais [19], ou ainda, quanto mais próximo for o qui-quadrado reduzido $\left(\chi_{\text {red }}^{2}\right)$ de 1, uma vez que:

$$
\chi_{\text {red }}^{2} \equiv \frac{\chi_{\text {mín. }}^{2}}{\nu} .
$$

Tendo encontrado os valores de melhor ajuste para os parâmetros, ou seja, aqueles que resultam no $\chi_{\text {mín. ou, }}^{2}$ equivalentemente, os que fornecem o ponto de máximo de $L\left(L_{\max .}=C e^{-\frac{1}{2} \chi_{\min }^{2}}\right)$, nos resta saber as regiões de confiança estatística para eles. Tais regiões são definidas por 19

$$
\chi_{\beta}^{2}=\chi_{\text {mín. }}^{2}+\Delta \chi^{2}(m, \beta),
$$

onde, $\Delta \chi^{2}$ representa o valor que delimita a região $\beta$ de confiança estatística. Na Tabela 2 são mostrados os valores de $\Delta \chi^{2}$ que definem as três regiões de confiança estatística mais utilizadas.

Em geral, os modelos cosmológicos possuem dois ou mais parâmetros. Para analisar cada um deles separadamente, faz-se uso de uma técnica chamada de marginalização, que consiste na integração da função densidade de probabilidade sobre todos os valores possível do

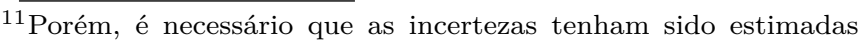
corretamente.
}

Tabela 2: Os valores de $\Delta \chi^{2}(m, \beta)$ que definem as regiões de confiança estatística.

\begin{tabular}{c|ccc}
\hline$\beta$ & $m=1$ & $m=2$ & $m=3$ \\
\hline $68,3 \%(1 \sigma)$ & 1,00 & 2,30 & 3,53 \\
$95,4 \%(2 \sigma)$ & 4,00 & 6,17 & 8,02 \\
$99,7 \%(3 \sigma)$ & 9,00 & 11,8 & 14,2 \\
\hline
\end{tabular}

parâmetro indesejado. Por exemplo, suponha uma função densidade de probabilidade $L\left(\alpha_{1}, \alpha_{2}\right)$ de dois parâmetros $\alpha_{1}$ e $\alpha_{2}$, a função densidade de probabilidade marginalizada do parâmetro $\alpha_{1}$, será dada por:

$$
L\left(\alpha_{1}\right)=\int L\left(\alpha_{1}, \alpha_{2}\right) d \alpha_{2} .
$$

Por fim, note que substituindo a equação 39 na (37), têm-se

$$
L\left(\Delta \chi^{2}\right)=L_{\max } e^{-\frac{1}{2} \Delta \chi^{2}} .
$$

Isso implica que para um único parâmetro, a região de $1 \sigma$ de confiança estatística é dada pela largura total de $L$ na altura

$$
L=L_{\text {max. }} e^{-\frac{1}{2}} \cong 0,6065 L_{\max } .
$$

já a região de $2 \sigma$ é dada pela largura total de $L$ na altura

$$
L=L_{\text {max. }} e^{-2} \cong 0,1353 L_{\text {max. }},
$$

e assim por diante (ver Figura 8).

\subsection{Oscilações Acústicas dos Bárions}

O Universo primordial foi muito quente e denso. Houve um período em que os fótons estavam acoplados com os bárions formando um único fluido ionizado. Nesse mesmo período aglomerados de matéria escura já começavam a se formar e atrair a matéria bariônica para seu interior através da atração gravitacional. No entanto, a pressão da radiação (fótons) sobre os bárions impedia a aglutinação e produziam ondas acústicas neste fluido (fóton-bárion), as quais hoje dá-se o nome de Oscilações Acústicas dos Bárions (BAO - do inglês Baryon Acoustic Oscillations).

Com a expansão do Universo, a densidade e temperatura desse fluido diminuíram, permitindo que os fótons desacoplem da matéria bariônica, passando assim a se propagar livremente. Logo, como a pressão dos fótons sobre os bárions deixa de existir, é de se esperar um "congelamento"na distribuição de bárions com um excesso de densidade em regiões próximas ao horizonte acústicd ${ }^{12}$ dessa onda. A Figura 4 ilustra de forma exagerada como os remanescentes desse fenômeno manifestam-se na distribuição atual dos diversos objetos astronômicos. Uma boa analogia deste processo são as ondas geradas na superfície de um lago ao cair sobre ele pingos de chuva. Se esse lago, com suas ondas concêntricas geradas pelos pingos de chuva subitamente congelasse, teríamos registradas na superfície gelada do lago as ondas produzidas.

\footnotetext{
12 Distância percorrida por essa onda acústica do início da perturbação até o desacoplamento.
} 


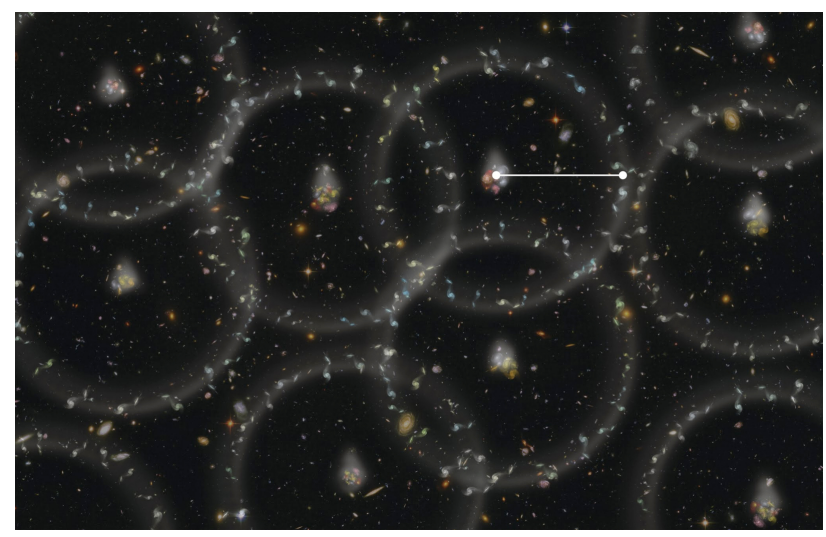

Figura 4: Concepção artística produzido pelo projeto BOSS (Baryon Oscillation Spectroscopic Survey) mostrando as esferas de bárions (galáxias) em torno dos aglomerados iniciais de matéria escura. As galáxias possuem uma ligeira tendência a se alinhar ao longo das bordas das esferas. O traço branco representa o raio do horizonte acústico. Crédito: Zosia Rostomian, Lawrence Berkeley National Laboratory.

Excesso de densidade como o mencionado pode ser mensurável através da função de correlação $\xi(s)$. A função de correlação mede se há desvios da distribuição espacial aleatória das galáxias. Em uma distribuição puramente aleatória, a probabilidade $d P$ de achar uma galáxia no volume $d V_{1}$ e uma outra no volume $d V_{2}$ é $d P=n^{2} d V_{1} d V_{2}$, onde $n$ é a densidade numérica das galáxias. Desvios do comportamento aleatório introduzem uma correção

$$
d P=n^{2} d V_{1} d V_{2}[1+\xi(s)]
$$

que define a mencionada função de correlação [21]. Portanto, se em uma separação espacial específica, o valor de $\xi(s)$ é positivo, indica que nessa separação existe uma maior probabilidade de se encontrar uma outra galáxia, visto que há um excesso de galáxias nessa separação, oposto a isso, valores negativos de $\xi(s)$ indicam um $d e$ ficit delas, logo uma menor probabilidade, já valores nulos, indicam uma distribuição aleatória (homogênea e uniforme).

A partir de um levantamento abrangendo uma região de $0,72 h^{-3} \mathrm{Gpc}^{3}$, com uma amostra de 46.748 galáxias vermelhas luminosas em $0,16<z<0,47$, o SDSS (Sloan Digital Sky Survey) construiu uma função de correlação de larga escala que mostra um pico bem definido em uma separação comóve ${ }^{13}$ de $100 h^{-1} \mathrm{Mpc}$ 22], como mostra a Figura 5 Isso implica que, dada uma galáxia formada em um pico de densidade, existe uma probabilidade maior de se encontrar outra galáxia a uma distância de $100 h^{-1} \mathrm{Mpc}$, que por sua vez, é aproximadamente igual ao horizonte acústico na época da recombinação $\left(r_{s} \approx 150 \mathrm{Mpc}\right)$. Note que, a função de correlação possui um valor maior em separações comóveis inferiores a $50 h^{-1} \mathrm{Mpc}$, isso ocorre

\footnotetext{
${ }^{13}$ Separação (ou distância) entre as galáxias medida em coordenadas comóveis, ou seja, em um sistema de coordenadas que acompanha a expansão do Universo.
}

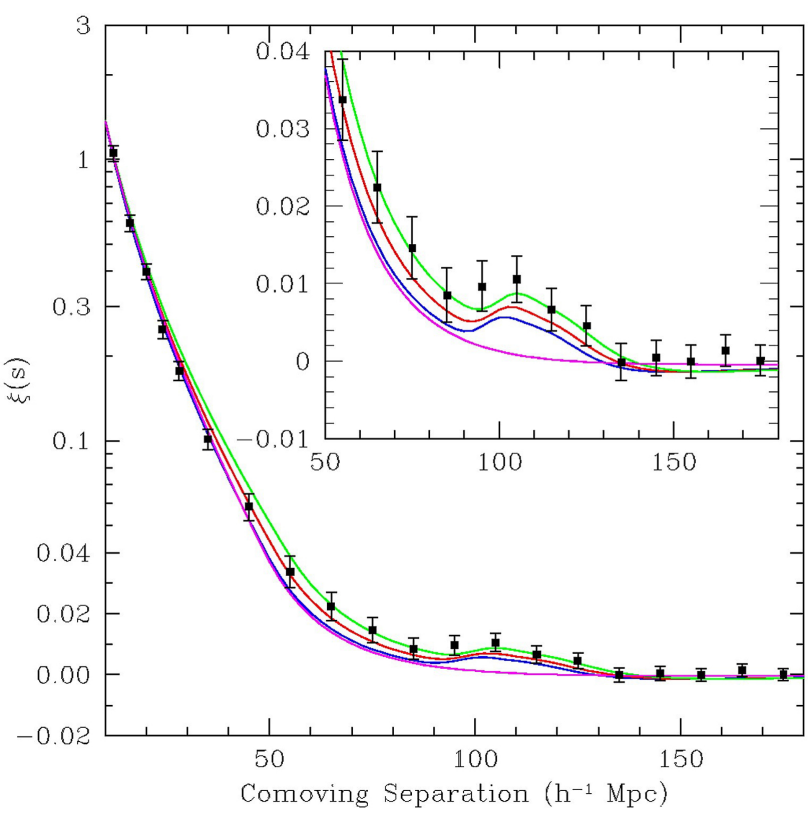

Figura 5: O pico acústico bariônico na função de correlação $\xi(s)$. As linhas coloridas representam modelos com diferentes valores para $\Omega_{0, C D M} h^{2}, 0,12$ (linha verde), 0,13 (linha vermelha) e 0,14 (linha azul), todos com $\Omega_{0, b} h^{2}=0,024$ e com um leve pico acústico em $100 h^{-1} \mathrm{Mpc}$, com exceção da linha magenta que representa um modelo CDM puro (sem matéria bariônica). (extraído de 22]).

devido a ação da força gravitacional, que atrai as estruturas para o pico de densidade, fazendo com que exista um excesso de probabilidade de encontrar uma galaxia mais próxima do pico.

O levantamento realizado pelo SDSS $[22]$ obteve um pico das $\mathrm{BAO}$ em $z_{*} \approx 0,35$. Para um Universo sem curvatura espacial, esse pico pode ser descrito por um parâmetro adimensional $A$, definido por D. J. Eisenstein et al. 22. como sendo,

$$
A\left(\mathbf{p} ; z_{*}\right) \equiv \frac{\sqrt{\Omega_{0, m}}}{E\left(\mathbf{p} ; z_{*}\right)^{1 / 3}}\left(\frac{1}{z_{*}} \int_{0}^{z_{*}} \frac{d z}{E(\mathbf{p} ; z)}\right)^{\frac{2}{3}} .
$$

Para $z_{*} \approx 0,35$ eles obtiveram $A(\mathbf{p} ; 0,35)=0,469 \pm$ 0,017 .

Atualmente existem medidas do pico das BAO em diferentes desvios para o vermelho, um exemplo que se destaca são as medidas obtidas da função de correlação do conjunto final de dados do WiggleZ Dark Energy Survey em um desvio para o vermelho $z_{*}=0,44,0,60$ e 0,73 , que resultaram em $A(0,44)=0,474 \pm 0,034, A(0,60)=$ $0,442 \pm 0,020$ e $A(0,73)=0,424 \pm 0,021$ [23]. Para simplificar o cálculo numérico realizado neste trabalho, utilizou-se apenas uma das medidas do parâmetro $A\left(z_{*}\right)$, a medida obtida pelo levantamento SDSS em $z_{*}=0,35$.

\subsection{Dados observacionais de $H(z)$}

O Parâmetro de Hubble, como já definido anteriormente, expressa a taxa com que o Universo se expande em 
um dado z. Este parâmetro carrega em si muitas características do modelo cosmológico. Na literatura é muito comum encontrá-lo expresso como sendo $H(z)=$ $H_{0} E(\mathbf{p} ; z)$. No caso específico do modelo $\Lambda$ CDM plano (ver equação 33 toma a seguinte forma:

$$
H(z)=H_{0} \sqrt{\Omega_{0, m}(1+z)^{3}+\left(1-\Omega_{0, m}\right)} .
$$

A determinação de $H(z)$ em diferentes pontos da evolução do Universo pode nos ajudar a melhor entender a dinâmica de sua expansão, além disso, tem se mostrado bastante útil na realização de testes cosmológicos, como o desenvolvido neste trabalho. Na Tabela 3 são apresentada as 18 medidas de $H(z)$ usadas nas nossas análises.

Existem hoje diferentes métodos para medir $H(z)$. O mais conhecidos deles é o que utiliza diferença de idade de galáxias [28,29], desenvolvido por R. Jimenez e A. Loeb 28]. Eles realizaram as primeiras medidas de $H(z)$ usando medições de diferença de idade $(\Delta t)$ entre duas galáxias que evoluíram passivamente e se formaram ao mesmo tempo, mas que estavam separadas por um pequeno intervalo de desvio para o vermelho $(\Delta z)$. As quantidades medidas estão relacionadas com o Parâmetro de Hubble pela seguinte relaçã ${ }^{14}$

$$
H(z)=-\frac{1}{(1+z)} \frac{d z}{d t}
$$

haja vista que pode-se inferir a derivada $d z / d t$ a partir da relação $\Delta z / \Delta t 28$.

\section{Resultados}

Nesta seção, usando o Método Qui-quadrado no âmbito do Modelo $\Lambda$ CDM Plano, são apresentadas as estimativas dos parâmetros $H_{0}, \Omega_{0, m}$ e alguns outros correlacionados.

\subsection{Análise estatística individual}

A análise estatística utilizando o BAO é realizada a partir do seguinte $\chi^{2}$ :

$$
\chi_{B A O}^{2}=\left(\frac{A\left(\mathbf{p} ; z_{*}\right)-0,469}{0,017}\right)^{2},
$$

\begin{tabular}{|c|c|c|c|c|c|c|c|}
\hline$z$ & $H(z)$ & $\sigma_{H(z)}$ & Ref. & $z$ & $H(z)$ & $\sigma_{H(z)}$ & Ref. \\
\hline 0,090 & 69 & 12 & 24 & 0,352 & 83 & 14 & 26 \\
\hline 0,170 & 83 & 8 & 25 & 0,593 & 104 & 13 & $\overline{26}$ \\
\hline 0,270 & 77 & 14 & 25 & 0,680 & 92 & 8 & $\overline{26}$ \\
\hline 0,400 & 95 & 17 & 25 & 0,781 & 105 & 12 & $\overline{26}$ \\
\hline 1,750 & 202 & 40 & 25 & 0,875 & 125 & 17 & 26 \\
\hline 1,300 & 168 & 17 & 25 & 1,037 & 154 & 20 & 26 \\
\hline 1,430 & 177 & 18 & 25 & 0,179 & 75 & 4 & 26 \\
\hline 1,530 & 140 & 14 & 25 & 0,199 & 75 & 5 & 26 \\
\hline 0,880 & 90 & 40 & 27 & 0,480 & 97 & 62 & 27 \\
\hline
\end{tabular}

Tabela 3: Dados observacionais de $H(z)$. A unidade das grandezas $H(z)$ e $\sigma_{H(z)}$ estão em $\mathrm{km} \mathrm{s}^{-1} \mathrm{Mpc}^{-1}$.

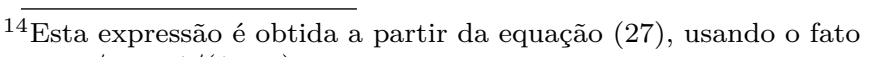
que $a / a_{0}=1 /(1+z)$. onde $A\left(\mathbf{p} ; z_{*}\right)$ é a expressão teórica, dada pela equação (45), 0, 469 é o valor observacional de $A$ para um $z=0,35$ e 0,017 é a incerteza associada a medida do $A$.

A partir do $\chi_{B A O}^{2}$ são gerados os contornos no plano $h-\Omega_{0, m}$ mostrado na Figura 6 (à esquerda), onde as regiões cinzas da mais escura para a mais clara correspondem as regiões de confiança estatística de $1 \sigma, 2 \sigma$ e $3 \sigma$, respectivamente. Deste plano, nota-se que o BAO sozinho não restringe o valor $h$ (ou equivalentemente $\left.H_{0}\right)$. Isso já era esperado, uma vez que o $A(\mathbf{p} ; z)$ é independente de $H_{0}$. Por outro lado, esta análise apresenta fortes restrições ao valor de $\Omega_{0, m}$, com o melhor ajuste em $\Omega_{0, m}=0,270$, que equivale a um $\Omega_{0, \Lambda}=0,730$. No entanto, usou-se aqui um único dado observacional, logo a análise usando apenas o BAO não possui significado estatístico relevante. Mas pode fornecer informações de regiões descartáveis em uma análise conjunta com outros observáveis, como por exemplo, os DOH(z). Neste contexto o uso do BAO torna-se bastante relevante.

Já a análise estatística utilizando os $\operatorname{DO} H(z)$, é realizada tendo como $\chi^{2}$, a expressão:

$$
\chi_{H(z)}^{2}=\sum_{i=1}^{N}\left(\frac{H\left(\mathbf{p} ; z_{i}\right)^{\text {teórico }}-H_{i}^{\text {obs }}}{\sigma_{i}^{\text {obs }}}\right)^{2},
$$

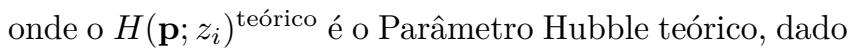
pela equação 46), $H_{i}^{\text {obs }}$ representa o valor observacional de $H(z)$ e $\sigma_{i}^{\text {obs }}$, a incerteza associada a medida de $H_{i}^{\text {obs }}$.

A Figura 6 (à direita) mostra os contornos no plano $h-\Omega_{0, m}$ gerados pelo $\chi_{H(z)}^{2}$, onde as regiões cinzas da mais escura para a mais clara correspondem as regiões de confiança estatística de $1 \sigma, 2 \sigma$ e $3 \sigma$, respectivamente. Ao contrário da análise com o BAO, esta apresenta significativa restrição ao valor de $h$ e uma não tão relevante ao valor do $\Omega_{0, m}$. Nesta análise, são obtidos os seguintes valores: $h=0,689_{-0,049}^{+0,051}, \Omega_{0, m}=0,317_{+0,103}^{-0,082}$ e um $\chi_{\text {red }}^{2}=0,73$, em $1 \sigma$ de confiança estatística.

Além disso, a partir desses resultados pode-se estimar, usando as equações 32 , (34) e (35), o $\Omega_{0, \Lambda}$, a idade total do Universo e o seu parâmetro de desaceleração, respectivamente. Nesse sentido, obtêm-se: $\Omega_{0, \Lambda}=0,683_{+0,103}^{-0,082}$ ( $1 \sigma$ de confiança estatística) e, no melhor ajuste, $t_{0}=$ 13,48 Ganos e $q_{0}=-0,524$.

\subsection{Análise estatística conjunta: $\mathrm{DO} H(z)+$ BAO}

A análise estatística conjunta é realizada a partir do seguinte $\chi^{2}$ :

$$
\begin{aligned}
\chi_{H(z)+B A O}^{2}= & \sum_{i=1}^{N}\left(\frac{H\left(\mathbf{p} ; z_{i}\right)^{\text {teórico }}-H_{i}^{\text {obs }}}{\sigma_{i}^{\text {obs }}}\right)^{2} \\
& +\left(\frac{A\left(\mathbf{p} ; z_{*}\right)-0,469}{0,017}\right)^{2}
\end{aligned}
$$

a partir dessa expressão, são produzidos os contornos (preenchidos com cores cinzas) no plano $h-\Omega_{0, m}$, 

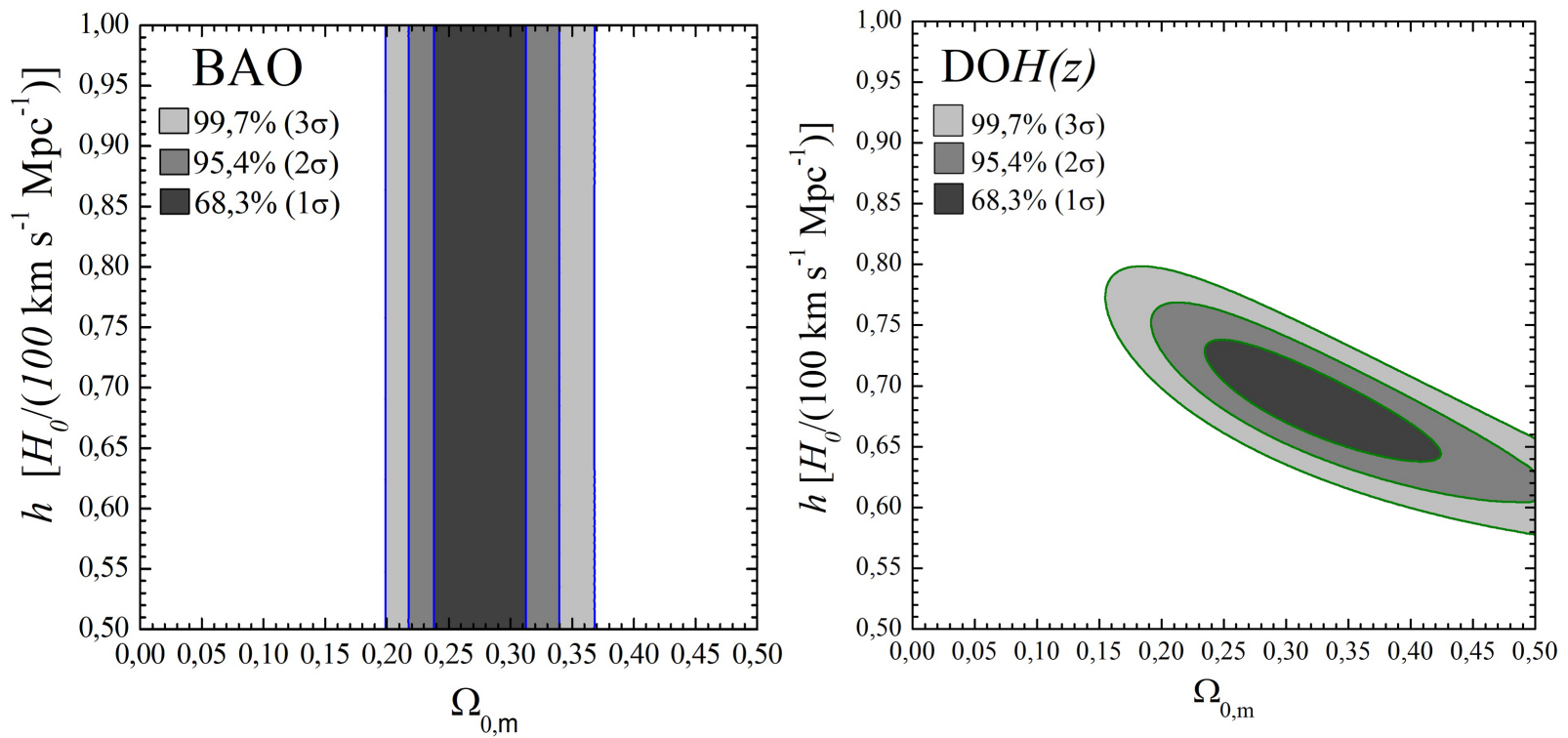

Figura 6: Contornos no plano $h-\Omega_{0, m}$ gerados pela estatística de qui-quadrado. Esquerda: referente a análise usando as Oscilações Acústicas dos Bárions (BAO). Direita: referente a análise usando os Dados Observacionais de $H(z)$ (DOH(z)). As regiões cinzas da mais escura para a mais clara correspondem as regiões de confiança estatística de $1 \sigma, 2 \sigma$ e $3 \sigma$, respectivamente.

exibidos na Figura 7, onde novamente, da parte mais escura para a mais clara, temos as regiões de confiança estatística de $1 \sigma, 2 \sigma$ e $3 \sigma$, respectivamente. Como bem mostra a Figura 7, a análise conjunta mostra-se bem eficaz, uma vez que a análise com o BAO (linhas tracejadas azuis) complementa a análise usando os $\mathrm{DO} H(z)$ (linhas tracejadas verdes) ao restringir o $\Omega_{0, m}$ de forma mais efetiva. Os resultados obtidos foram: $h=0,709_{-0,038}^{+0,029}$ e $\Omega_{0, m}=0,280_{+0,040}^{-0,030}$ em $1 \sigma$ de confiança estatística e $h=0,709_{-0,057}^{+0,051}$ e $\Omega_{0, m}=0,280_{+0,060}^{-0,050}$ em $2 \sigma$ de confiança estatística, com um $\chi_{\text {red }}^{2}=0,73$. Mais uma vez, usando os valores citados de $h$ e $\Omega_{0, m}$ obtêm-se um $\Omega_{0, \Lambda}=0,720_{+0,040}^{-0,030}(1 \sigma$ de confiança estatística) e, no melhor ajuste, $t_{0}=13,56$ Ganos e $q_{0}=-0,580$.

A Figura 8 mostra a função densidade de probabilidade marginalizada do parâmetro $h$ (figura à esquerda) e do $\Omega_{0, m}$ (figura à direita). As linhas horizontais são os cortes nas regiões de $1 \sigma(68,3 \%)$ e $2 \sigma(95,4 \%)$ de confiança estatística. Para se obter a função para o $h$, marginaliza-se sobre o parâmetro $\Omega_{0, m} \mathrm{e}$, da mesma forma, para se obter a função para o $\Omega_{0, m}$, marginaliza-se sobre o parâmetro $h$. As estimativas obtidas para o $h$, ou equivalentemente $H_{0}$ (ver equação 2) e o $\Omega_{0, m}$ são exibidas na Tabela 4. A partir dessas estimativas, usando a propagação de incertezas, pode-se estimar também os parâmetros $\Omega_{0, \Lambda}$, $q_{0}$ e $t_{0}$, cujos resultados na região de $1 \sigma$ de confiança estatística são: $\Omega_{0, \Lambda}=0,720 \pm 0,022, t_{0}=13,62 \pm 0,50$ Ganos e $q_{0}=-0,580 \pm 0,033$.

É importante elucidar que em todas as análises realizadas neste trabalho, não foram considerados erros sistemáticos. Segundo D. Stern et al. 27], a diferença de idade relativa, um dos métodos empregado para obter as medidas de $H(z)$, possui de 2 a $3 \%$ de erros sis-

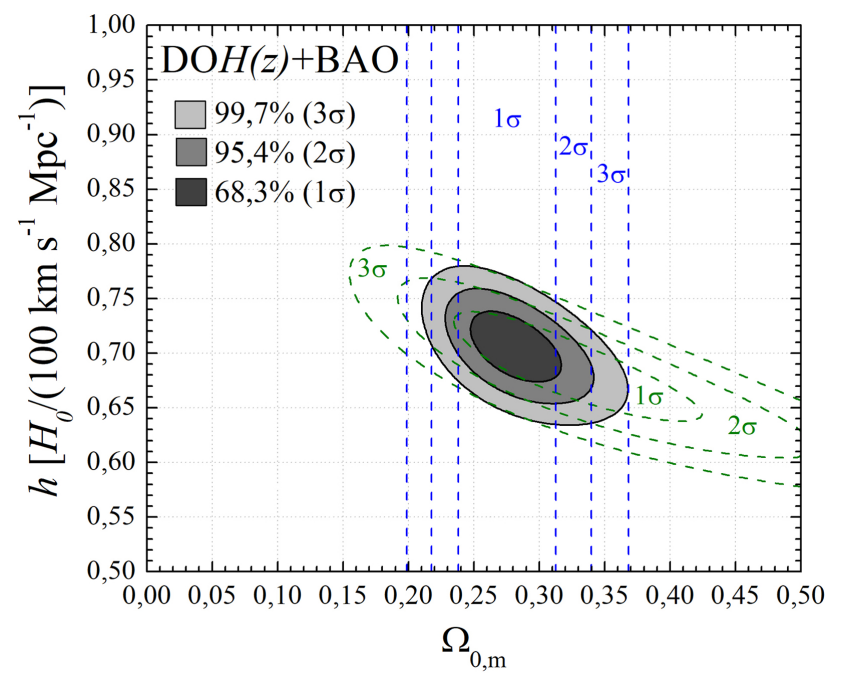

Figura 7: Contornos no plano $h-\Omega_{0, m}$ gerados pela estatística de qui-quadrado. Os contornos preenchidos nas cores cinzas são referentes a análise conjunta dos $\mathrm{DO} H(z)$ mais $\mathrm{BAO}$, os contornos representados pelas linhas verdes tracejadas são referentes a análise usando somente os $\mathrm{DO} H(z)$, já os representados pelas linhas azuis tracejadas são referentes à análise usando somente ao BAO.

Tabela 4: Estimativas dos parâmetros $H_{0}\left(\mathrm{~km} \mathrm{~s}^{-1} \mathrm{Mpc}^{-1}\right)$, $\Omega_{0, m}$ e $\Omega_{0, \Lambda}$, referentes a análise conjunta.

\begin{tabular}{c|cc}
\hline $\mathrm{DO} H(z)+\mathrm{BAO}$ & $H_{0}$ & $\Omega_{0, m}$ \\
\hline $1 \sigma(68,3 \%)$ & $70,6 \pm 2,1$ & $0,280 \pm 0,022$ \\
$2 \sigma(95,4 \%)$ & $70,6_{-4,1}^{+4,2}$ & $0,280_{-0,042}^{+0,049}$ \\
\hline$\chi_{\text {red }}^{2}$ & 0,75 & 0,75 \\
\hline
\end{tabular}



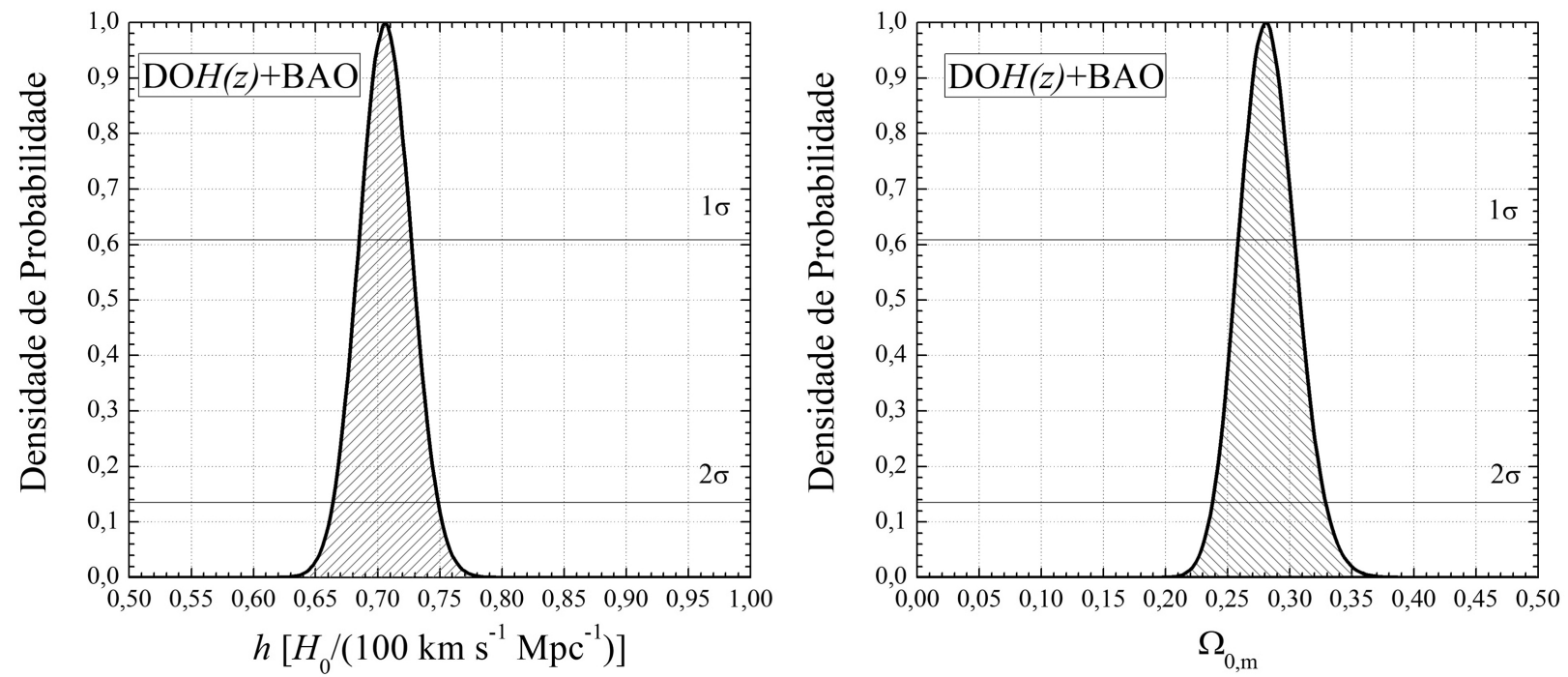

Figura 8: Função de densidade de probabilidade do parâmetro $h$ (à esquerda) e $\Omega_{0, m}$ (à direita), obtidas a partir da análise conjunta. As duas linhas horizontais são os cortes nas regiões de $1 \sigma$ e $2 \sigma$ de confiança estatística.

temáticos. Ao incluir 3\% de erros sistemáticos em quadratura $\left(\sigma_{\text {total }}^{2}=\sigma_{\text {est }}^{2}+\sigma_{\text {sit }}^{2}\right)$ para todas as medidas de $H(z)$, obtêm-se a partir da análise conjunta $(\mathrm{DOH}(z)+\mathrm{BAO})$ : $h=0,710 \pm 0,030$ e $\Omega_{0, m}=0,280 \pm 0,031$ em $1 \sigma$ de confiança estatística. Logo, vê-se que a inclusão dos erros sistemáticos nas análises não altera significativamente os valores de melhor ajuste, no entanto, produz um aumento das incertezas.

\section{Comparação com os resultados do Planck e do WMAP}

A Radiação Cósmica de Fundo (RCF) é sem dúvida uma das maiores e mais importantes fontes de informação contemporânea para o estudo do Universo, sendo assim, crucial para o desenvolvimento da Cosmologia. Ao longo dos anos, vários experimentos foram desenvolvidos para estudar acuradamente a RCF. Os de maior destaque foram: o satélite COBE (Cosmic Background Explorer), lançado em 1989, o satélite WMAP (Wilkinson Microwave Anisotropy Probe), lançado pela NASA em 2001 e o satélite espacial Planck lançado pela Agência Espacial Europeia (ESA - European Space Agency), em 14 de maio de 2009.

A partir dos dados obtidos por estes satélites, é possível estimar com bastante precisão os mais diversos parâmetros cosmológicos, inclusive todos os abordados neste artigo.
Na tabela 5 são apresentadas as estimativas obtidas por dois grandes grupos, uma referente a análise dos dados de nove anos do WMAP [17] e a outra dos dados do Planck 18], além das obtidas neste trabalho (expostas na Tabela 4), todas na região de $1 \sigma$ de confiança estatística. Como se pode ver, as estimativas realizadas neste artigo, usando a análise conjunta dos $\operatorname{DO} H(z)$ e o BAO, além de serem bastantes restritivas, são dentro da região de confiança estatística, compatíveis com as obtidas pelos dois grupos já citados.

\section{Conclusão}

Neste artigo, além de abordar e discutir alguns conceitos e fundamentos teóricos da Cosmologia Moderna, foi realizada uma análise estatística, usando os $\mathrm{DOH}(z)$ e o BAO em um modelo $\Lambda$ CDM Plano, capaz de estimar de forma significativa os valores dos seguintes parâmetros cosmológicos: $H_{0}, \Omega_{0, m}, \Omega_{0, \Lambda}, t_{0}$ e $q_{0}$.

Dos resultados apresentados, conclui-se que a melhor e mais restritiva estimativa é obtida através da análise conjunta dos dois observáveis, $\mathrm{DO} H(z)$ e BAO (a Figura 7 ilustra bem esta afirmação). Na análise conjunta, ao marginalizar sobre o parâmetro $\Omega_{0, m}$, foi obtido um $H_{0}=70,6 \pm 2,1 \mathrm{~km} \mathrm{~s}^{-1} \mathrm{Mpc}^{-1}$, em $1 \sigma$ de confiança estatística e da mesma forma, ao marginalizar sobre o parâmetro $h$, foi obtido um $\Omega_{0, m}=0,280 \pm 0,022$, em $1 \sigma$

Tabela 5: Três estimativas diferentes para os parâmetros $H_{0}, \Omega_{0, m}, \Omega_{0, \Lambda}, t_{0}$ e $q_{0}$, referentes a análise conjunta dos DOH( $\left.z\right)$ e $\mathrm{BAO}$, aos dados do WMAP e do Planck, respectivamente. As estimativas com o simbolo $\dagger$ foram obtidos usando a propagação de incerteza. Todas estão na região de $1 \sigma$ de confiança estatística.

\begin{tabular}{c|ccccc}
\hline Estimativas & $H_{0}\left(\mathrm{~km} \mathrm{~s}^{-1} \mathrm{Mpc}^{-1}\right)$ & $\Omega_{0, m}$ & $\Omega_{0, \Lambda}$ & $t_{0}(\mathrm{G}$ anos $)$ & $q_{0}$ \\
\hline DOH $(z)+\mathrm{BAO}$ & $70,6 \pm 2,1$ & $0,280 \pm 0,022$ & $0,720 \pm 0,022^{\dagger}$ & $13,62 \pm 0,50^{\dagger}$ & $-0,580 \pm 0,033^{\dagger}$ \\
WMAP 9 anos 2013 17. & $70,0 \pm 2,20$ & $0,279 \pm 0,0231$ & $0,721 \pm 0,025$ & $13,74 \pm 0,11$ & $-0,581 \pm 0,027^{\dagger}$ \\
Colaboração Planck 2016 18. & $67,81 \pm 0,92$ & $0,308 \pm 0,012$ & $0,692 \pm 0,012$ & $13,799 \pm 0,038$ & $-0,538 \pm 0,013^{\dagger}$ \\
\hline
\end{tabular}


de confiança estatística (ver Figura 8). Usando estas duas medidas e a propagação de incertezas em um modelo $\Lambda \mathrm{CDM}$ plano, estimou-se um $\Omega_{0, \Lambda}=0,720 \pm 0,022$, $t_{0}=13,62 \pm 0,50$ Ganos e um $q_{0}=-0,580 \pm 0,033$, na região de $1 \sigma$ de confiança estatística.

Por meio das definições detalhadas dos parâmetros cosmológicos e da descrição didática e objetiva dos princípios envolvidos na análise estatística, espera-se que mesmos os leitores sem afinidade com tais técnicas compreendam e até possam fazer uso desse método de estimativa de parâmetros cosmológicos.

Por fim, comparando as estimativas realizadas neste artigo com as desenvolvidas por dois grandes grupos, o WMAP 17] e a Colaboração Planck [18], conclui-se que, apesar da simplicidade e dos poucos dados usados, as estimativas aqui apresentadas, em $1 \sigma$ de confiança estatística, estão em ótimo acordo com as apresentadas por estes dois grupos, como bem mostra a Tabela 5.

\section{Agradecimentos}

Agradeço ao Conselho Nacional de Desenvolvimento Científico e Tecnológico ( $\mathrm{CNPq}$ ) e ao Departamento de Física Teórica e Experimental da Universidade Federal do Rio Grande do Norte (DFTE-UFRN) pelo suporte financeiro a esse projeto. Gostaria de agradecer também ao amigo Gesiel Rodrigues da S. Neto e a minha esposa Esther G. Jácome Lino, pela leitura e as várias sugestões.

\section{Referências}

[1] I. Waga, Rev. Bras. Ensino Fís. 27, 157 (2005).

[2] E.P. Hubble, Proc. Natl. Acad. Sci. U.S.A. 15, 168 (1929).

[3] E.P. Hubble and M.L. Humason, The Astrophysical Journal 74, 43 (1931).

[4] I. Waga, Rev. Bras. Ensino Fís. 22, 163 (2000).

[5] G.B. Lima Neto, Astronomia Extragaláctica - Notas de Aula (Departamento de Física, Universidade de São Paulo, 2016).

[6] V. Mukhanov, Physical Foundations of Cosmology (Cambridge University Press, Oxford, 2005).

[7] M. Carmeli, Classical Fields: General Relativity and Gauge Theory (J. Wiley, New York, 1982), p. 650.

[8] D. Soares, Rev. Bras. Ensino Fís. 35, 3302 (2013).

[9] M.P. Hobson, G.P. Efstathiou and A.N. Lasenby, General Relativity: An Introduction for Physicists (Cambridge University Press, Cambridge, 2006), p. 572.

[10] A. Liddle, An Introduction to Modern Cosmology (J. Wiley, New York, 2003), 2nd ed.

[11] K.K.S. Wu, O. Lahav and M.J. Rees, Nature 397, 225 (1999).

[12] M.I. Scrimgeour, T. Davis, C. Blake, J.B. James, G.B. Poole, L. Staveley-Smith, S. Brough, M. Colless, C. Contreras, W. Couch, et al., Monthly Notices of the Royal Astronomical Society 425, 116 (2012).

[13] S. Weinberg, Cosmology (Oxford University Press, Oxford, 2008), p. 593.
[14] B. Ryden, Introduction to Cosmology (Cambridge University Press, Oxford, 2016), p. 276.

[15] R.J.A. Lambourne, Relativity, Gravitation and Cosmology (Cambridge University Press, Oxford, 2010), p. 307.

[16] E. Harrison, The Astrophysical Journal 403, 28 (1993).

[17] G. Hinshaw, D. Larson, E. Komatsu, D.N. Spergel, C.L. Bennett, J. Dunkley, M.R. Nolta, M. Halpern, R.S. Hill, N. Odegard, et al., The Astrophysical Journal Supplement Series 208, 19, (2013).

[18] P.A.R. Ade, N. Aghanim, M. Arnaud, M. Ashdown, J. Aumont, C. Baccigalupi, A.J. Banday, R.B. Barreiro, J.G. Bartlett, N. Bartolo, et al., Astronomy \& Astrophysics 594, A13 (2016).

[19] J.V. Wall and C.R. Jenkins, Practical Statistics for Astronomers (Cambridge University Press, Oxford, 2012), p. 353.

[20] J.H. Vuolo, Fundamentos da Teoria de Erros (Blucher, São Paulo, 1996), p. 249.

[21] J.E. Horvath, G. Lugones, M.P. Allen, S. Scarano Júnior e R. Teixeira, Cosmologia Física: Do Micro ao Macro Cosmos e Vice-Versa (Editora Livraria da Fisica, São Paulo, 2007), p. 298.

[22] D.J. Eisenstein, I. Zehavi, D.W. Hogg, R. Scoccimarro, M.R. Blanton, R.C. Nichol, R. Scranton, H. Seo, M. Tegmark, Z. Zheng, et al., The Astrophysical Journal 633, 560 (2005).

[23] C. Blake, E. Kazin, F. Beutler, T.M. Davis, D. Parkinson, S. Brough, M. Colless, C. Contreras, W. Couch, S. Croom, et al., Monthly Notices of the Royal Astronomical Society 418, 1707 (2011).

[24] R. Jimenez, L. Verde, T. Treu and D. Stern, The Astrophysical Journal 593, 622 (2003).

[25] J. Simon, L. Verde and R. Jimenez, Phys. Rev. D. 71, 123001 (2005).

[26] M. Moresco, A. Cimatti, R. Jimenez, L. Pozzetti, G. Zamorani, M. Bolzonella, J. Dunlop, F. Lamareille, M. Mignoli, H. Pearce, et al., Journal of Cosmology and Astroparticle Physics 2012, 006 (2012).

[27] D. Stern, R. Jimenez, L. Verde, M. Kamionkowski and S.A. Stanford, Journal of Cosmology and Astroparticle Physics 2010, 8 (2010).

[28] R. Jimenez and A. Loeb, The Astrophysical Journal 573, 37 (2002).

[29] C. Zhang, H. Zhang, S. Yuan, S. Liu, T. Zhang and Y. Sun, Research in Astronomy and Astrophysics 14, 1221 (2014). 